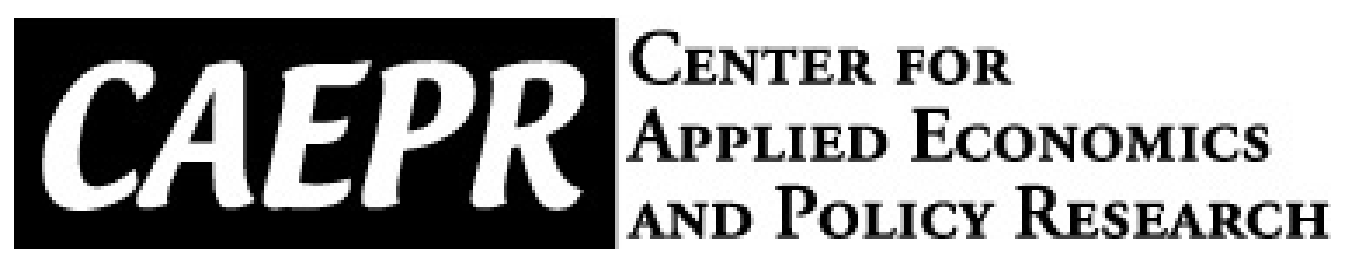

CAEPR Working Paper

\#008-2010

\title{
Modelling Overnight and Daytime Returns Using a Multivariate GARCH-Copula Model
}

\author{
Long Kang \\ THE OPTIONS CLEARING CORPORATION \\ (email: lkang@theocc.com) \\ Simon H Babbs \\ THE OPTIONS ClEARING CORPORATION \\ (email: sbabbs@theocc.com)
}

\section{June 2010}

This paper can be downloaded without charge from the Social Science Research Network electronic library at: http://ssrn.com/abstract=1710799.

The Center for Applied Economics and Policy Research resides in the Department of Economics at Indiana University Bloomington. CAEPR can be found on the Internet at:

http://www.indiana.edu/ caepr. CAEPR can be reached via email at caepr@indiana.edu or via phone at 812-855-4050.

(02008 by NAME. All rights reserved. Short sections of text, not to exceed two paragraphs, may be quoted without explicit permission provided that full credit, including $\odot$ notice, is given to the source. 


\title{
Modelling Overnight and Daytime Returns Using a Multivariate GARCH-Copula Model $^{1}$
}

\section{Long Kang}

THE OPTIONS CLEARING CORPORATION

(email: lkang@ theocc.com)

\section{Simon H Babbs}

THE OPTIONS CLEARING CORPORATION

(email: sbabbs@theocc.com)

\begin{abstract}
We introduce a multivariate GARCH-Copula model to describe joint dynamics of overnight and daytime returns for multiple assets. The conditional mean and variance of individual overnight and daytime returns depend on their previous realizations through a variant of GARCH specification, and two Student's $t$ copulas describe joint distributions of both returns respectively. We employ both constant and time-varying correlation matrices for the $t$ copulas and with the time-varying case the dependence structure of both returns depends on their previous dependence structures through a DCC specification. We estimate the model by a two-step procedure, where marginal distributions are estimated in the first step and copulas in the second. We apply our model to overnight and daytime returns of SPDR ETFs of nine major sectors and briefly illustrate its use in risk management and asset allocation. Our empirical results show higher mean, lower variance, fatter tails and lower correlations for overnight returns than daytime returns. Daytime returns are significantly negatively correlated with previous overnight returns. Moreover, daytime returns depend on previous overnight returns in both conditional variance and correlation matrix (through a DCC specification). Most of our empirical findings are consistent with the asymmetric information argument in the market microstructure literature. With respect to econometric modelling, our results show a DCC specification for correlation matrices of $t$ copulas significantly improves the fit of data and enables the model to account for time-varying dependence structure.
\end{abstract}

JEL classification: C32, G12, G14.

Key words: Overnight and daytime returns, GARCH-Copula models.

\section{Introduction}

Modeling the dynamics of overnight and daytime returns is important in at least two aspects. First, a good description of overnight and daytime returns can help to test alternative theories on different features of market microstructure during the day and night. Second, from a practitioner's point of view, many financial instruments (such as index options) are based on the opening prices of underlying assets. Thus, it is necessary to distinguish between overnight and daytime returns for risk management or asset allocation purposes. There is plenty of work documenting different empirical properties of overnight and daytime returns. Some show that

\footnotetext{
${ }^{1}$ Views expressed in this paper are those of the authors and not necessarily those of any organization to which the authors are affiliated.
} 
overnight returns are statistically higher than daytime returns, while others show higher returns over trading periods than non-trading periods (see Cliff et al. (2008) and Keim \& Stambaugh (1984)). Some show that daytime returns are statistically negatively correlated with previous overnight returns, while others show that the two returns are largely independent (see Gallo et al. (2001), Branch \& Ma (2006) and Oldfield \& Rogalski (1980)). The variance of daytime returns is significantly higher than that of overnight returns (see French \& Roll (1986) and Lockwood \& Linn (1990)). Overnight returns are more leptokurtic than daytime returns ${ }^{2}$ (see Masulis \& $\mathrm{Ng}$ (1995)). Those empirical patterns also generate interest in proposing theoretical models to explain them (see Admati \& Pfleiderer $(1988,1989)$ and Hong \& Wang (2000)).

To the best of our knowledge, current literature on this topic only deals with the univariate case and there is no work on modeling the joint dynamics of multiple assets. Our work attempts to fill this gap. First, we introduce a comprehensive model which can reasonably well capture key empirical aspects of both returns and can be easily implemented by practitioners. Second, by applying the model to the data, we expect to find some different features of the dependence structure between both returns. Moreover, we illustrate how the model can be effectively used for risk management or asset allocation purposes.

With a GARCH-Copula framework, we can more flexibly construct the joint distribution of multiple returns. The dynamics of overnight and daytime returns for each asset are described by a GARCH process, where the conditional mean and variance depend on the previous realizations of both returns and innovations are described by Student's $t$ distributions. Then we use two Student's $t$ copulas to link overnight and daytime returns of multiple assets respectively. The constant correlation matrices in Student's $t$ copulas are assumed and estimated first, and then to describe the time-varying feature of dependence structure we implement a DCC (Dynamic Conditional Correlation) specification for Student's $t$ copulas. We estimate the model by a twostep procedure, where the marginal distributions are estimated in the first step and the copulas in the second.

We apply our model to the overnight and daytime returns of SPDR ETFs of nine major sectors between March 11, 2003 and July 19, 2007. At the individual level, overnight returns have statistically significant positive average returns while daytime returns depend on previous overnight returns negatively. The conditional variance of daytime returns is consistently higher than that of overnight returns. Almost all the ETFs have lower DoF parameters for overnight returns than for daytime returns, which is consistent with the observed higher kurtosis of overnight returns. In addition to their own lags, the conditional variances of overnight (daytime) returns has some degree of dependence on previous daytime (overnight) returns.

With constant Student's $t$ copulas, overnight returns have lower values of correlation matrix and DoF parameters than daytime returns. Time-varying $t$ copulas yield similar results. Overnight returns tend to be less dependent on each other than daytime returns. Tail dependence patterns are mixed for both returns. Moreover, time-varying copula models show that daytime returns depend on previous overnight returns. This adds to observed non-linear dependence between

${ }^{2}$ See Kang \& Babbs (2010b) for a comprehensive empirical investigation. 
daytime and previous overnight returns. We believe most of our empirical findings are consistent with the asymmetric information argument in theoretical models such as Admati \& Pfleiderer $(1988,1989)$. Moreover, our results show that a DCC specification for correlation matrices of $t$ copulas significantly improves fit of data and enables the model to account for time-varying dependence structure.

The rest of the paper is organized as follows. Section 2 introduces the multivariate GARCHCopula model and its estimation and simulation procedures. Section 3 applies the model to the overnight and daytime returns of SPDR ETFs of nine sectors. Section 4 briefly illustrates the application of the model for risk management and asset allocation purposes. Section 5 concludes.

\section{A multivariate GARCH-Copula model}

\subsection{Individual returns}

We model individual returns using a variant of GARCH specification. Let $R_{i, t}=\left[r_{i, n, t} r_{i, d, t}\right]^{\prime}$ be overnight and daytime returns for asset $i$ observed at the open and close respectively on day $t$. The individual returns for asset $i$ are

$$
r_{i, n, 0}, r_{i, d, 0}, \ldots, r_{i, n, t-1}, r_{i, d, t-1}, r_{i, n, t}, r_{i, d, t}, \ldots r_{i, n, T}, r_{i, d, T}
$$

We specify the conditional mean as

$$
\begin{aligned}
& r_{i, n, t}=\alpha_{i, 0}+\alpha_{i, 1} r_{i, d, t-1}+\alpha_{i, 2} r_{i, n, t-1}+\eta_{i, n, t}, \\
& r_{i, d, t}=\beta_{i, 0}+\beta_{i, 1} r_{i, n, t}+\beta_{i, 2} r_{i, d, t-1}+\eta_{i, d, t} .
\end{aligned}
$$

where $r_{i, n, t}$ depends on previous daytime return $r_{i, d, t-1}$ and previous overnight return $r_{i, n, t-1}$, and similarly $r_{i, d, t}$ depends on $r_{i, n, t}$ and $r_{i, d, t-1}$. We specify residuals as

$$
\begin{aligned}
& \eta_{i, n, t}=\sqrt{h_{i, n, t}} \varepsilon_{i, n, t}, \\
& \eta_{i, d, t}=\sqrt{h_{i, d, t}} \varepsilon_{i, d, t},
\end{aligned}
$$

where $\varepsilon_{i, n, t}$ and $\varepsilon_{i, d, t}$ are i.i.d. innovations with zero mean and unitary variance respectively and are independent between each other at all times, and $h_{i, n, t}$ and $h_{i, d, t}$ are conditional variance described by the following equations.

$$
\begin{aligned}
& h_{i, n, t}=\theta_{i, 0}+\theta_{i, 1} \eta_{i, d, t-1}^{2}+\theta_{i, 2} \eta_{i, n, t-1}^{2}+\theta_{i, 3} h_{i, n, t-1}, \\
& h_{i, d, t}=\delta_{i, 0}+\delta_{i, 1} \eta_{i, n, t}^{2}+\delta_{i, 2} \eta_{i, d, t-1}^{2}+\delta_{i, 3} h_{i, d, t-1} .
\end{aligned}
$$


where $\theta_{i, j}>0$ and $\delta_{i, j}>0$ for $j=0, \ldots, 3, \theta_{i, 2}+\theta_{i, 3}<1$ and $\delta_{i, 2}+\delta_{i, 3}<1$. Those parameter restrictions guarantee conditional variances are always non-negative and squared residuals are stationary $^{3}$. We assume that the innovations $\varepsilon_{i, n, t}$ and $\varepsilon_{i, d, t}$ have standardized Student's $t$ distribution as

$$
\varepsilon_{i, n, t} \sim S T\left(0,1, v_{i, n}\right), \varepsilon_{i, d, t} \sim S T\left(0,1, v_{i, d}\right)
$$

where $v_{i, n}$ and $v_{i, d}$ are degree-of-freedom (DoF) parameters and we have $v_{i, n}, v_{i, d}>2$ to ensure the existence of second movements.

It is worth noting that we can use a more sophisticated specification for marginal distributions of individual returns. For instance, we can include more explanatory variables in equations (1) and (2) to possibly better describe the conditional mean, include a GJR specification (see Glosten et al (1993)) in equations (5) and (6) to account for the asymmetric effect of stock returns on the conditional variance and specify a distribution with time-varying high-moment parameters (e.g. time-varying Hansen's (1994) skewed $t$ distribution) for residual innovations. Nevertheless, we will focus more on modelling joint distributions here. As we believe the current setup describes the data reasonably well, we will leave those options for further research.

\subsection{Copulas}

After specifying marginal distributions of returns, we need two copula functions to link overnight and daytime returns respectively across all assets. To formulate the joint distribution of returns for $N$ assets, we are facing the following multiple time series,

$$
R_{1,0}, \ldots, R_{N, 0}, \ldots, R_{1, t-1}, \ldots, R_{N, t-1}, R_{1, t}, \ldots, R_{N, t}, \ldots, R_{1, T}, \ldots, R_{N, T}
$$

Let $F_{t}, F_{d, t}$ and $F_{n, t}$ be the conditional cumulative distribution function (c.d.f.) for $R_{1, t}, \ldots, R_{N, t}$, $r_{1, d, t}, \ldots, r_{N, d, t}$, and $r_{1, n, t}, \ldots, r_{N, n, t}$. At each time $t$, the conditional distribution of $R_{1, t}, \ldots, R_{N, t}$ is given as

$$
F_{t}\left(R_{1, t}, \ldots, R_{N, t} \mid R_{1, t-1}, \ldots, R_{N, t-1}, \ldots, R_{1,0}, \ldots, R_{N, 0}\right)=F_{d, t}\left(r_{1, d, t}, \ldots, r_{N, d, t} \mid \Omega_{d, t-1}\right) F_{n, t}\left(r_{1, n, t}, \ldots, r_{N, n, t} \mid \Omega_{n, t-1}\right),(7)
$$

where $\Omega_{d, t-1}=\left\{r_{1, n, t}, \ldots, r_{N, n, t}, r_{1, d, t-1}, \ldots, r_{N, d, t-1}, \ldots, r_{1, d, 0}, \ldots, r_{N, d, 0}, r_{1, n, 0}, \ldots, r_{N, n, 0}\right\}$ and $\Omega_{n, t-1}=\left\{r_{1, d, t-1}, \ldots\right.$, $\left.r_{N, d, t-1}, r_{1, n, t-1}, \ldots, r_{N, n, t-1}, \ldots, r_{1, d, 0}, \ldots, r_{N, d, 0}, r_{1, n, 0}, \ldots, r_{N, n, 0}\right\}$. Let $F_{i, d, t}$ and $F_{i, n, t}$, for $i=1, \ldots, N$, be the conditional c.d.f.'s for $r_{i, d, t}$ and $r_{i, n, t}$. Then the conditional joint distributions $F_{d, t}\left(r_{1, d, t}, \ldots, r_{N, d, t} \mid \Omega_{d, t-1}\right)$ and $F_{n, t}\left(r_{1, n, t}, \ldots, r_{N, n, t} \mid \Omega_{n, t-1}\right)$ can be modelled using two copulas as,

\footnotetext{
${ }^{3}$ A more general VARMA model for the vector of squared overnight and daytime returns can be proposed. The parameter restrictions will depend on the stationartiy and identification of the corresponding VARMA process. See Kang \& Babbs (2010a).
} 


$$
\begin{gathered}
F\left(r_{1, d, t}, \ldots, r_{N, d, t} \mid \Omega_{d, t-1}\right)=C_{d}\left(F\left(r_{1, d, t} \mid \Omega_{d, t-1}\right), \ldots, F\left(r_{N, d, t} \mid \Omega_{d, t-1}\right) \mid \Omega_{d, t-1}\right), \\
F\left(r_{1, n, t}, \ldots, r_{N, n, t} \mid \Omega_{n, t-1}\right)=C_{n}\left(F\left(r_{1, n, t} \mid \Omega_{n, t-1}\right), \ldots, F\left(r_{N, n, t} \mid \Omega_{n, t-1}\right) \mid \Omega_{n, t-1}\right),
\end{gathered}
$$

where $C_{n}$ and $C_{d}$ are the two copula $c . d . f$.'s. It is worth noting that the copula function and marginal distributions are all conditional on the previous information set.

There are many choices of copula functions for modelling the dependence structure of multiple variables. We use Student's $t$ copula in this paper. The $c . d$.f. of Student's $t$ copula is given by

$$
C\left(u_{1}, \ldots, u_{N}\right)=T_{R, v}\left(T_{v}^{-1}\left(u_{1}\right), \ldots, T_{v}^{-1}\left(u_{N}\right)\right),
$$

where $T_{R, v}$ is the $N$-dimensional Student's $t$ distribution with correlation matrix $R$ and DoF parameter $v$, and $T_{v}^{-1}(\cdot)$ is the inverse of univariate standard Student's $t$ distribution ${ }^{4}$. The probability density function (p.d.f.) of Student's $t$ copula is

$$
c\left(u_{1}, \ldots, u_{N}\right)=\frac{t_{R, v}\left(T_{v}^{-1}\left(u_{1}\right), \ldots, T_{v}^{-1}\left(u_{N}\right)\right)}{\prod_{i=1}^{N} t_{v}\left(T_{v}^{-1}\left(u_{i}\right)\right)},
$$

where $t_{R, v}(\cdot)$ is the density function of $T_{R, v}$ and $t_{v}(\cdot)$ is the density of $T_{v}$, the standard Student's $t$ distribution.

When using Student's $t$ copulas for both returns, we can assign constant or time-varying correlation matrices for both $t$ copulas. As for the time-varying $t$ copulas, we borrow the idea of DCC-GARCH models to make the two sets of correlation matrices depend on past realizations. Let $\varsigma_{c n, t}=\left(T_{v_{c n}}^{-1}\left(u_{1, n, t}\right), \ldots, T_{v_{c n}}^{-1}\left(u_{N, n, t}\right)\right)^{\prime}$ and $\varsigma_{c d, t}=\left(T_{v_{c d}}^{-1}\left(u_{1, d, t}\right), \ldots, T_{v_{c d}}^{-1}\left(u_{N, d, t}\right)\right)^{\prime}$ according to (8) and $Q_{n, t}$ and $Q_{d, t}$ be the conditional covariance matrices of $\varsigma_{c n, t}$ and $\zeta_{c d, t}$ respectively. The timevarying correlation matrices $R_{c n, t}$ and $R_{c d, t}$ of $t$ copulas are governed by the dynamics of $Q_{n, t}$ and $Q_{d, t}$ as

$$
\begin{aligned}
Q_{n, t} & =\Pi_{0}+\pi_{1}\left(\varsigma_{c d, t-1} \varsigma_{c d, t-1}^{\prime}\right)+\pi_{2}\left(\varsigma_{c n, t-1} \varsigma_{c n, t-1}^{\prime}\right)+\pi_{3} Q_{d, t-1}+\pi_{4} Q_{n, t-1}, \\
Q_{d, t} & =\Psi_{0}+\psi_{1}\left(\varsigma_{c n, t} \varsigma_{c n, t}^{\prime}\right)+\psi_{2}\left(\varsigma_{c d, t-1} \varsigma_{c d, t-1}^{\prime}\right)+\psi_{3} Q_{n, t}+\psi_{4} Q_{d, t-1},
\end{aligned}
$$

where $\pi_{i} \geq 0$ and $\psi_{i} \geq 0$ for $i=1, \ldots, 4$, and $\pi_{2}+\pi_{4}<1$ and $\psi_{2}+\psi_{4}<1$ to ensure the system of (9) and (10) is valid and stationary. With stationarity, it can be shown that

\footnotetext{
${ }^{4}$ In contrast to the previous standardized Student's $t$ distribution, the standard Student's $t$ distribution here has variance as $v /(v-2)$.
} 


$$
\begin{aligned}
& \Pi_{0}=\left(1-\left(\pi_{2}+\pi_{4}\right)\right) S_{n}-\left(\pi_{1}+\pi_{3}\right) S_{d}, \\
& \Psi_{0}=\left(1-\left(\psi_{2}+\psi_{4}\right)\right) S_{d}-\left(\psi_{1}+\psi_{3}\right) S_{n},
\end{aligned}
$$

where $S_{n}$ and $S_{d}$ are the unconditional covariance of $\zeta_{c n, t}$ and $\zeta_{c d, t}{ }^{5}$. Let $q_{i, j, n, t}$ and $q_{i, j, d, t}$ be the $i, j$-element of $Q_{n, t}$ and $Q_{d, t}$ respectively, then the $i, j$-elements of $R_{c n, t}$ and $R_{c d, t}$ are given as

$$
\begin{aligned}
& \rho_{i, j, n, t}=\frac{q_{i, j, n, t}}{\sqrt{q_{i, i, n, t} q_{j, j, n, t}}}, \\
& \rho_{i, j, d, t}=\frac{q_{i, j, d, t}}{\sqrt{q_{i, i, d, t} q_{j, j, d, t}}} .
\end{aligned}
$$

With $Q_{n, 0}=S_{n}$ and $Q_{d, 0}=S_{d}$, the equations (9) to (14) completely govern the dynamics of the correlations matrices $R_{c n, t}$ and $R_{c d, t}$. For the correlation matrices to be positive definite, we have the following sufficient conditions.

Proposition 1 In equations (9) to (14), if

a) $\pi_{i} \geq 0$ and $\psi_{i} \geq 0$ for $i=1, \ldots, 4$,

b) $\pi_{2}+\pi_{4}<1$ and $\psi_{2}+\psi_{4}<1$,

c) all eigenvalues of $S_{n}$ and $S_{d}$ are strictly positive,

d) all eigenvalues of $\Pi_{0}$ and $\Psi_{0}$ are strictly positive,

then the correlations matrices $R_{c n, t}$ and $R_{c d, t}$ are positive definite.

Proof: First, a) and b) guarantee the system is stationary and $S_{n}$ and $S_{d}$ exist. c) guarantees $Q_{n, 0}$ and $Q_{d, 0}$ are positive definite. With a) and d), $Q_{n, t}$ and $Q_{d, t}$ are the sum of positive semi-definite and positive definite matrices with non-negative coefficients and therefore are positive definite for all $t$. Based on the proposition 1 in Engle \& Sheppard (2001), we prove that $R_{c n, t}$ and $R_{c d, t}$ are positive definite.

\subsection{Estimation}

We estimate the whole density function by ML estimation procedures. Let $F$ and $F_{0}$ be the c.d.f.'s for $R_{1,0}, \ldots, R_{N, 0}, \ldots, R_{1, T}, \ldots, R_{N, T}$ and $R_{1,0}, \ldots, R_{N, 0}$. Using Bayes' Theorem, the whole joint distribution of returns can be written as

\footnotetext{
${ }^{5}$ See Appendix for derivation details.
} 


$$
\begin{gathered}
F\left(R_{1,0}, \ldots, R_{N, 0}, \ldots, R_{1, T}, \ldots, R_{N, T}\right)= \\
F_{0}\left(R_{1,0}, \ldots, R_{N, 0}\right) \prod_{t=1}^{T}\left(C_{d}\left(F_{1, d, t}\left(r_{1, d, t} \mid \Omega_{d, t-1}\right), \ldots, F_{N, d, t}\left(r_{N, d, t} \mid \Omega_{d, t-1}\right) \mid \Omega_{d, t-1}\right)\right. \\
\left.\cdot C_{n}\left(F_{1, n, t}\left(r_{1, n, t} \mid \Omega_{n, t-1}\right), \ldots, F_{N, n, t}\left(r_{N, n, t} \mid \Omega_{n, t-1}\right) \mid \Omega_{n, t-1}\right)\right) .
\end{gathered}
$$

Correspondingly, the joint density of returns can be written as

$$
\begin{aligned}
f\left(R_{1,0}, \ldots, R_{N, 0}, \ldots, R_{1, T}, \ldots, R_{N, T}\right)= & f_{0}\left(R_{1,0}, \ldots, R_{N, 0}\right) \prod_{t=1}^{T}\left(c_{d}\left(F_{1, d, t}\left(r_{1, d, t}\right), \ldots, F_{N, d, t}\left(r_{N, d, t}\right)\right) \prod_{i=1}^{N} f_{i, d, t}\left(r_{i, d, t}\right)\right. \\
& \left.\cdot c_{n}\left(F_{1, n, t}\left(r_{1, n, t}\right), \ldots, F_{N, n, t}\left(r_{N, n, t}\right)\right) \prod_{i=1}^{N} f_{i, n, t}\left(r_{1, n, t}\right)\right),
\end{aligned}
$$

where $\Omega_{d, t-1}$ and $\Omega_{n, t-1}$ are suppressed for notation convenience, $f_{0}, f_{i, d, t}$ and $f_{i, n, t}$ are the densities for $F_{0}, F_{i, d, t}$ and $F_{i, n, t}$, and $c_{n}$ and $c_{d}$ are the two copula densities for overnight and daytime returns respectively. From equation (15), we can continue to write the density as

$$
\begin{aligned}
f\left(R_{1,0}, \ldots, R_{N, 0}, \ldots, R_{1, T}, \ldots, R_{N, T}\right)= & f_{0}\left(R_{1,0}, \ldots, R_{N, 0}\right) \prod_{t=1}^{T}\left(c_{d}\left(F_{\varepsilon_{1, d, t}}\left(\varepsilon_{1, d, t}\right), \ldots, F_{\varepsilon_{N, d, t}}\left(\varepsilon_{N, d, t}\right)\right) \prod_{i=1}^{N} f_{\varepsilon_{i, d, t}}\left(\varepsilon_{i, d, t}\right) \frac{1}{\sqrt{h_{i, d, t}}}\right. \\
& \left.c_{n}\left(F_{\varepsilon_{1, n, t}}\left(\varepsilon_{1, n, t}\right), \ldots, F_{\varepsilon_{N, n, t}}\left(\varepsilon_{N, n, t}\right)\right) \prod_{i=1}^{N} f_{\varepsilon_{i, n, t}}\left(\varepsilon_{i, n, t}\right) \frac{1}{\sqrt{h_{i, n, t}}}\right),
\end{aligned}
$$

where $F_{\varepsilon_{i, d, t}}$ and $F_{\varepsilon_{i, n, t}}$ are the c.d.f.'s for $\varepsilon_{i, d, t}$ and $\varepsilon_{i, n, t}$, and $f_{\varepsilon_{i, d, t}}$ and $f_{\varepsilon_{i, n, t}}$ are corresponding densities.

Let $\Theta=\left\{\theta_{c n}, \theta_{c d}, \theta_{1}, \ldots, \theta_{N}\right\}$ be a set of parameters for the two copula densities $c_{n}$ and $c_{d}$, and $R_{i, t}$ for $i=1, \ldots, N$ respectively. Omitting the first term $f_{0}\left(R_{1,0}, \ldots, R_{N, 0}\right)$, we can write the $\log$ likelihood as

$$
\begin{aligned}
L(\Theta) & =\sum_{t=1}^{T} \log c_{d}\left(F_{\varepsilon_{1, d, t}}\left(\varepsilon_{1, d, t}\right), \ldots, F_{\varepsilon_{N, d, t}}\left(\varepsilon_{N, d, t}\right)\right)+\sum_{t=1}^{T} \log c_{n}\left(F_{\varepsilon_{1, n, t}}\left(\varepsilon_{1, n, t}\right), \ldots, F_{\varepsilon_{N, n, t}}\left(\varepsilon_{N, n, t}\right)\right) \\
& +\sum_{i=1}^{N} \sum_{t=1}^{T} \log f_{\varepsilon_{i, d, t}}\left(\varepsilon_{i, d, t}\right) \frac{1}{\sqrt{h_{i, d, t}}}+\sum_{i=1}^{N} \sum_{t=1}^{T} \log f_{\varepsilon_{i, n, t}}\left(\varepsilon_{i, n, t}\right) \frac{1}{\sqrt{h_{i, n, t}}} .
\end{aligned}
$$

To estimate all the parameters simultaneously often leads to convergence problem of maximizing (17). Therefore, we maximize the whole log-likehihood by a two-step procedure. First, we estimate the marginal distribution of each asset. For asset $i$, the log-likelihood is

$$
L\left(\theta_{i}\right)=\sum_{t=1}^{T} \log f_{\varepsilon_{i, d, t}}\left(\varepsilon_{i, d, t}\right) \frac{1}{\sqrt{h_{i, d, t}}}+\sum_{t=1}^{T} \log f_{\varepsilon_{i, n, t}}\left(\varepsilon_{i, n, t}\right) \frac{1}{\sqrt{h_{i, n, t}}} .
$$


To further facilitate the estimation, we first estimate the conditional mean in equations (1) and (2) by ordinary least squares (OLS). Then with estimated OLS residuals, we can estimate the variance equations (5) and (6) by maximizing (18). The specific log-likehood becomes

$$
\begin{aligned}
L= & \sum_{t=1}^{T} \log f_{S T}\left(\frac{r_{i, n, t}-\hat{\alpha}_{i, 0}-\hat{\alpha}_{i, 1} r_{i, d, t-1}-\hat{\alpha}_{i, 2} r_{i, n, t-1}}{\sqrt{h_{i, n, t}}} \mid v_{i, n}\right)+\sum_{t=1}^{T} \log f_{S T}\left(\frac{r_{i, d, t}-\hat{\beta}_{i, 0}-\hat{\beta}_{i, 1} r_{i, n, t}-\hat{\beta}_{i, 2} r_{i, d, t-1}}{\sqrt{h_{i, d, t}}} \mid v_{i, d}\right) \\
& +\sum_{t=1}^{T} \log \frac{1}{\sqrt{h_{i, n, t}}}+\sum_{t=1}^{T} \log \frac{1}{\sqrt{h_{i, d, t}}},
\end{aligned}
$$

where $f_{S T}$ denotes the density of standardized Student's $t$ distribution. With all the marginal distributions being estimated, the only component left out in (17) is the copula part. With estimated marginal distribution parameters, we can estimate the two copulas by maximizing

$$
L\left(\theta_{c n}, \theta_{c d}\right)=\sum_{t=1}^{T} \log c_{d}\left(\hat{F}\left(\varepsilon_{1, d, t}\right), \ldots, \hat{F}\left(\varepsilon_{N, d, t}\right)\right)+\sum_{t=1}^{T} \log c_{n}\left(\hat{F}\left(\varepsilon_{1, n, t}\right), \ldots, \hat{F}\left(\varepsilon_{N, n, t}\right)\right),
$$

where $\hat{F}(\cdot)$ is the estimated $c . d . f$. for each innovation. Whether the maximization of (19) is easy or not depends on the specific copula functions. With normal copulas, we can derive analytical ML estimates very easily. With Student's $t$ copulas, however, the parameters $\theta_{c n}$ and $\theta_{c d}$ consist of correlation matrices $R_{c n}$ and $R_{c d}$, and the DoF parameters $v_{c n}$ and $v_{c d}$, and there is no easy analytical solution for maximizing (19). To smoothly solve this maximization problem, with $\zeta_{c n, t}=\left(T_{v_{c n}}^{-1}\left(u_{1, n, t}\right), \ldots, T_{v_{c n}}^{-1}\left(u_{N, n, t}\right)\right)^{\prime}$ and $\zeta_{c d, t}=\left(T_{v_{c d}}^{-1}\left(u_{1, d, t}\right), \ldots, T_{v_{c d}}^{-1}\left(u_{N, d, t}\right)\right)^{\prime}$, we assign $\hat{R}_{c n}$ and $\hat{R}_{c d}$ as the sample correlation matrices of $\zeta_{c n, t}$ and $\zeta_{c d, t} . \hat{R}_{c n}$ and $\hat{R}_{c d}$ are functions of DoF parameters $v_{c n}$ and $v_{c d}$. Therefore, we can plug $\hat{R}_{c n}$ and $\hat{R}_{c d}$ into (19) and solve the maximization problem in terms of $v_{c n}$ and $v_{c d}$. Furthermore, as parameters for the two copulas are separate, we can maximize the two components in (19) separately to solve for $v_{c n}$ and $v_{c d}$ respectively.

To estimate the time-varying $t$ copula, we still maximize the log-likelihood as in (19) except that the time-varying $t$ copulas are used. To reduce the number of parameters to directly estimate, we express the time-varying correlation matrices $R_{c n, t}$ and $R_{c d, t}$ as functions of DoF parameters $v_{c n}$ and $v_{c d}$ as in the above constant copula case. Specifically, with given $v_{c n}$ and $v_{c d}$, we have the estimated unconditional covariance of $\zeta_{c n, t}$ and $\zeta_{c d, t}$ as $\hat{S}_{n}$ and $\hat{S}_{d}$, and we set the initial $Q_{n, 0}$ and $Q_{d, 0}$ equal to $\hat{S}_{n}$ and $\hat{S}_{d}$ respectively. Equations (9) to (14) completely describes the dynamics of the correlation matrices $R_{c n, t}$ and $R_{c d, t}$. Then all the parameters to estimate are $\pi_{i}$, $\omega_{i}$ for $i=1, \ldots, 4, v_{c n}$ and $v_{c d}$, and the maximization is conducted with the corresponding restrictions.

Generally, this two-step estimation procedure is called inference for the margins (IFM) method. Joe (1997) shows that under regular conditions the IFM estimator is consistent and has the property of asymptotic normality and Patton (2006) also shows that this two-step method yields asymptotically efficient and normal parameter estimates. 


\subsection{Density forecast and simulations}

With parameter estimates, we can forecast the joint density and then simulate future overnight and daytime returns. Specifically, with estimated parameters $\hat{\Theta}$ and $\Omega_{n, t-1}$, we can sequentially forecast $f\left(r_{1, n, t}, \ldots, r_{N, n, t} \mid \Omega_{n, t-1}\right), \quad f\left(r_{1, d, t}, \ldots, r_{N, d, t} \mid \Omega_{d, t-1}\right)$ and then $f\left(r_{1, d, t}, \ldots, r_{N, d, t}, r_{1, n, t}, \ldots, r_{N, n, t}\right.$ $\left.\mid \Omega_{n, t-1}\right)$. Accordingly, we can sequentially simulate $\left\{r_{1, n, t}, \ldots, r_{N, n, t}\right\}$ and $\left\{r_{1, d, t}, \ldots, r_{N, d, t}\right\}$. With $\hat{\theta}_{c n}$, we first simulate the copula $C_{n}$ to get a simulated vector $\left\{\tilde{u}_{1}, \ldots, \tilde{u}_{n}\right\}$. Using estimated GARCH parameters $\hat{\theta}_{1}, \ldots, \hat{\theta}_{N}$, and equations (1), (3) and (5), we can back out $\left\{\tilde{r}_{1, n, t}, \ldots, \tilde{r}_{N, n, t}\right\}$. Finally, we can use $\hat{\theta}_{c d}$ to simulate copula $C_{d}$ and then back out $\left\{\tilde{r}_{1, d, t}, \ldots, \tilde{r}_{N, d, t}\right\}$.

\section{An empirical investigation}

\subsection{Data}

We apply our model to returns of SPDR ETFs of nine sectors. The nine symbols are XLY, XLP, XLE, XLF, XLV, XLI, XLB, XLK, and XLU representing the sectors of Consumer Discretionary, Consumer Staples, Energy, Financial, Healthcare, Industrial, Materials, Technology and Utilities. Figure 1 plots the daily log returns of nine sector SPDR ETFs between from December 22, 1998 to November 17, 2008. The period from December 22, 1998 to March 10, 2003 features a high volatility due to burst internet bubbles and terrorist attacks, the second from March 11, 2003 to July 19, 2007 is of low volatility and the last one from July 20, 2007 to November 19, 2008 features an unprecedented high volatility due to the financial crisis. We choose the second period for our analysis. The open and close prices and dividend payments are directly downloaded from http://finance.yahoo.com/. We calculate log overnight and daytime returns based on the open and close prices and dividend payments.

Table 1 reports the descriptive statistics (mean, standard deviation, Skewness and Kurtosis) for overnight and daytime returns of the SPDR ETFs of nine sectors between March 11, 2003 and July 19, 2007. We find overnight returns are all positive and consistently higher than daytime returns. Daytime returns have significantly higher standard deviations than overnight returns. Skewness for both returns has mixed signs, kurtosis is greater than three for both returns and overnight returns tend to have higher kurtosis.

$<$ Insert Figure 1 and Table1 here.>

\subsection{Empirical results}

Table 2 reports the OLS estimates for the conditional mean equations for each asset. The values in italics are standard errors. Estimates in bold are statistically significant at a 5\% confidence level. Interestingly, the constant terms are all statistically significant and positive. Not many overnight returns significantly depend on previous daytime and overnight returns. For significant ones, the signs are mixed. In contrast, most constant terms for daytime returns are not statistically significant. Almost all daytime returns statistically depend on previous overnight returns negatively. Few of them depend on their own lag values. 
Table 3 reports the GARCH estimates of marginal distributions for individual assets. Almost all GARCH terms and DoF parameters are statistically significant, while ARCH terms have mixed statistical significance. Except XLI, we find all the other ETFs have lower DoF parameters for overnight returns than for daytime returns, which is consistent with the observed higher Kurtosis of overnight returns. Figure 2 plots the estimated conditional variance for the nine ETFs. The conditional variance of daytime returns is consistently higher than that of overnight returns.

Table 4 reports the estimates for the two constant Student's $t$ copulas governing the dependence structure of overnight and daytime returns respectively. Overnight returns have lower values of correlation matrix and DoF parameters than daytime returns. The higher correlation matrix of the $t$ copulas for daytime returns generally indicate that daytime returns are more correlated than overnight returns, even though correlation matrix in $t$ copulas seldom exactly equals to correlation matrix of underlying returns ${ }^{6}$. The lower value of DoF of $t$ copulas for overnight returns does not necessarily suggest fatter joint tails than daytime returns as overnight returns have larger correlation matrix. Similarly, the tail dependence coefficient (TDC) depends on both correlation matrix and DoF parameter of $t$ copulas.

Table 5 reports the estimates for the two time-varying Student's $t$ copulas. We first observe that the time-varying $t$ copula yields significantly higher log-likelihood than its constant case, indicating a better fit of data. Estimates of the parameters in equations (9) and (10) suggest the dependence structure of overnight returns has significant influence on that of the following daytime returns while the dependence of overnight returns appears to be mainly determined by its previous dependence structure. This observation adds to higher moment dependence between daytime and previous overnight returns in a multivariate setting. Similar with the constant copulas, overnight returns have lower DoF than daytime returns. Figure 3 plots time-varying conditional correlation parameters of the $t$ copulas for four selected ETF pairs. Correlation parameters for daytime returns consistently fluctuate above those for overnight returns. Figure 4 plots the conditional TDC for four selected pairs, where the comparison patterns are mixed.

In summary, our empirical results show that overnight returns have higher mean, lower variance and higher kurtosis than daytime returns. In terms of dependence structure, overnight returns generally have lower correlations than daytime returns. Moreover, daytime returns significantly depend on previous overnight returns in first and second univariate moments and dependence structure. We believe that most of our observations are consistent with the asymmetric information argument in theoretical work such as Admati \& Pfleiderer $(1988,1989)$.

$<$ Insert Tables 2, 3, 4 and 5, and Figures 2, 3 and 4 here.>

\footnotetext{
6 They are equal when DoF parameter of the marginal distribution of each asset equals to that of the copulas.
} 


\section{Applications}

\subsection{Risk management}

Facing overnight and daytime returns, risk managers monitor portfolios whose returns depend on both overnight and daytime returns of underlying assets. Specifically, at time $t-1$ and with an information set $\Omega_{n, t-1}$, risk managers are concerned with the return distribution of a portfolio at the end of time $t$. Let $P_{t}=g_{t}\left(r_{1, n, t}, \ldots, r_{N, n, t}, r_{1, d, t}, \ldots, r_{N, d, t}\right)$ be the portfolio return at end of time $t$, which is a function of both overnight and daytime returns of underlying assets at time $t$. From section 2.4, we can forecast $f\left(r_{1, d, t}, \ldots, r_{N, d, t}, r_{1, n, t}, \ldots, r_{N, n, t} \mid \Omega_{n, t-1}\right)$. Accordingly, the value-at-risk $(\mathrm{VaR})$ and expected shortfall (ES) can be calculated.

\subsection{Asset allocation}

As a simple example of asset allocation, investors solve a one-period investment problem, allocate their wealth among risk-free and risky assets at the beginning of each time period and maximize their expected utility. Let $W_{t}=w_{t}\left(r_{1, n, t}, \ldots, r_{N, n, t}, r_{1, d, t}, \ldots, r_{N, d, t}\right)$ be the wealth at time $t$ and with an information set $\Omega_{n, t-1}$, investors allocate their wealth to maximize their expected utility as

$$
\int \ldots \int U\left(W_{t}\right) f\left(r_{1, d, t}, \ldots, r_{N, d, t}, r_{1, n, t}, \ldots, r_{N, n, t} \mid \Omega_{n, t-1}\right) d r_{1, d, t} \ldots d r_{N, n, t},
$$

where $U(\cdot)$ is a certain utility function. Usually, the maximization problem is based on the numerical simulations of the expected utility.

\section{Conclusion}

We introduce a multivariate GARCH-Copula model to describe joint dynamics of both overnight and daytime returns of multiple assets. The conditional mean and variance of individual returns depend on their previous realizations, and two (constant and time-varying) Student's $t$ copulas link both returns respectively. We apply the model to SPDR ETFs of nine sectors and illustrate its use in risk management and asset allocation.

There are several possibilities for extensions. First, we can include more explanatory economic variables or factors in the system to better predict joint density. Second, we can investigate how to estimate the model by a Bayesian approach. Finally, we can test the model's performance in an out-of-sample manner with more financial applications. We leave those possibilities for future research. 


\section{Appendix}

Let $\mathrm{E}_{d, t-1}=\varsigma_{d, t-1} \varsigma_{d, t-1}^{\prime}$ and $\mathrm{E}_{n, t-1}=\varsigma_{n, t-1} \varsigma_{n, t-1}^{\prime}$, we can write equations (9) and (10) as

$$
\begin{aligned}
& \mathrm{E}_{n, t}=\Pi_{0}+\left(\pi_{1}+\pi_{3}\right) \mathrm{E}_{d, t-1}+\left(\pi_{2}+\pi_{4}\right) \mathrm{E}_{n, t-1}+V_{n, t}-\pi_{3} V_{d, t-1}-\pi_{4} V_{n, t-1}, \\
& \mathrm{E}_{d, t}=\Psi_{0}+\left(\psi_{1}+\psi_{3}\right) \mathrm{E}_{n, t}+\left(\psi_{2}+\psi_{4}\right) \mathrm{E}_{d, t-1}+V_{d, t}-\psi_{3} V_{n, t}-\psi_{4} V_{d, t-1}
\end{aligned}
$$

where

$$
\begin{aligned}
& V_{n, t}=\mathrm{E}_{n, t}-Q_{n, t}, \\
& V_{d, t}=\mathrm{E}_{d, t}-Q_{d, t} .
\end{aligned}
$$

We can write equations (20) and (21) in block matrices as

$$
\begin{aligned}
{\left[\begin{array}{cc}
1 & 0 \\
-\left(\psi_{1}+\psi_{3}\right) & 1
\end{array}\right]\left[\begin{array}{l}
\mathrm{E}_{n, t} \\
\mathrm{E}_{d, t}
\end{array}\right] } & =\left[\begin{array}{l}
\Pi_{0} \\
\Psi_{0}
\end{array}\right]+\left[\begin{array}{cc}
\pi_{2}+\pi_{4} & \pi_{1}+\pi_{3} \\
0 & \psi_{2}+\psi_{4}
\end{array}\right]\left[\begin{array}{l}
\mathrm{E}_{n, t-1} \\
\mathrm{E}_{d, t-1}
\end{array}\right]+\left[\begin{array}{cc}
1 & 0 \\
-\psi_{3} & 1
\end{array}\right]\left[\begin{array}{l}
V_{n, t} \\
V_{d, t}
\end{array}\right] \\
& +\left[\begin{array}{cc}
-\pi_{4} & -\pi_{3} \\
0 & -\psi_{4}
\end{array}\right]\left[\begin{array}{l}
V_{n, t-1} \\
V_{d, t-1}
\end{array}\right] .
\end{aligned}
$$

Accordingly, we have

$$
\begin{aligned}
{\left[\begin{array}{c}
\mathrm{E}_{n, t} \\
\mathrm{E}_{d, t}
\end{array}\right] } & =\left[\begin{array}{c}
\Pi_{0}+\left(\psi_{1}+\psi_{3}\right) \Psi_{0} \\
\Psi_{0}
\end{array}\right]+\left[\begin{array}{cc}
\pi_{2}+\pi_{4} & \left(\pi_{1}+\pi_{3}\right)+\left(\psi_{1}+\psi_{3}\right)\left(\psi_{2}+\psi_{4}\right) \\
0 & \psi_{2}+\psi_{4}
\end{array}\right]\left[\begin{array}{l}
\mathrm{E}_{n, t-1} \\
\mathrm{E}_{d, t-1}
\end{array}\right] \\
& +\left[\begin{array}{cc}
1-\psi_{3}\left(\psi_{1}+\psi_{3}\right) & \psi_{1}+\psi_{3} \\
-\psi_{3} & 1
\end{array}\right]\left[\begin{array}{l}
V_{n, t} \\
V_{d, t}
\end{array}\right]+\left[\begin{array}{cc}
-\pi_{4} & -\pi_{3}-\psi_{4}\left(\psi_{1}+\psi_{3}\right) \\
0 & -\psi_{4}
\end{array}\right]\left[\begin{array}{l}
V_{n, t-1} \\
V_{d, t-1}
\end{array}\right] .
\end{aligned}
$$

The above system is a $\operatorname{VARMA}(1,1)$ process and we require $\left|\pi_{2}+\pi_{4}\right|<1$ and $\left|\psi_{2}+\psi_{4}\right|<1$ for stationarity. Under stationarity, we can take expectations on both sides of (24) and with $E\left(V_{n, t}\right)=\mathbf{0}$ and $E\left(V_{d, t}\right)=\mathbf{0}$, we have

$$
\begin{aligned}
& \Pi_{0}=\left(1-\left(\pi_{2}+\pi_{4}\right)\right) S_{n}-\left(\pi_{1}+\pi_{3}\right) S_{d}, \\
& \Psi_{0}=\left(1-\left(\psi_{2}+\psi_{4}\right)\right) S_{d}-\left(\psi_{1}+\psi_{3}\right) S_{n},
\end{aligned}
$$

where $S_{n}$ and $S_{d}$ are the unconditional covariance of $\boldsymbol{\zeta}_{c n, t}$ and $\boldsymbol{\zeta}_{c d, t}$. 


\section{Reference}

Admati, Anat R, and Paul Pfleiderer. "A Theory of Intraday Patterns: Volume and Price Variability." Review of Financial Studies 1 (1988): 3-40.

Admati, Anat R, and Paul Pfleiderer. "Divide and Conquer: A Theory of Intraday and Day-ofthe-Week Mean Effects." Review of Financial Studies 2 (1989): 189-223.

Branch, Ben S, and Aixin Ma. "The Overnight Return, One More Anomaly." (Available at SSRN: http://ssrn.com/abstract=937997) 92006.

Cliff, Michael T, Michael J Cooper, and Huseyin Gulen. "Return Differences between Trading and Non-Trading Hours: Like Night and Day." Available at SSRN: http://ssrn.com/abstract=1004081, September 2008.

Engle, Robert F, and Kevin Sheppard. "Theoretical and Empirical Properties of Dynamic Conditional Correlation Multivariate GARCH." University of California at San Diego, Economics Working Paper Series, 2001.

French, Kenneth R, and Richard Roll. "Stock return variances: The arrival of information and the reaction of traders." Journal of Financial Economics 17 (1986): 5-26.

Gallo, Giampiero, Yongmiao Hong, and Tae-Hwy Lee. "Modeling the Impact of Overnight Surprises on Intra-daily Stock Returns." Proceedings for Business and Economic Statistics. American Statistical Association, 2001.

Glosten, Lawrence R, Ravi Jagannathan, and David E Runkle. "On the Relation between the Expected Value and the Volatility of the Nominal Excess Return on Stocks." Journal of Finance 48, no. 5 (1993): 1779-1801.

Hansen, Bruce E. "Autoregressive Conditional Density Estimation." International Economic Review 35, no. 3 (August 1994): 705-730.

Hong, Harrison, and Jiang Wang. "Trading and Returns under Periodic Market Closures." Journal of Finance 55 (2000): 297-354.

Joe, Harry. Multivariate Models and Dependence Concepts. London: Chapman and Hall, 1997.

Kang, Long, and Simon H Babbs. "Overnight and Daytime Returns: an Empirical Investigation." Working paper, 2010a.

Kang, Long, and Simon H Babbs. "Tail Dependence of Major U.S. Stocks." Edited by A Jalilvand and T Malliaris. Risk Management and Corporate Governance, 2010b, forthcoming.

Keim, Donald, and Robert Stambaugh. "A Further Investigation of Weekend Effect in Stock Returns." Journal of Finance 39 (1984): 819-835.

Lockwood, Larry J, and Scott C Linn. "An Examination of Stock Market Return Volatility during Overnight and Intraday Periods, 1964-1989." Journal of Finance 45 (1990): 591-601. 
Masulis, Ronald W, and Victor K Ng. "Overnight and Daytime Stock-Return Dynamics on the London Stock Exchange: the Impacts of "Big Bang" and the 1987 Stock-Market Crash." Journal of Business \& Economic Statistics 13, no. 4 (October 1995).

Oldfield, George S, and Richard J Rogalski. "A Theory of Common Stock Returns over Trading and Non-Trading Periods." Journal of Finance 35 (1980): 729-751.

Patton, Andrew J. "Estimation of Copula Models for Time Series of Possibly Different Lengths." Journal of Applied Econometrics 21, no. 2 (2006): 147-173. 
Figures and Tables
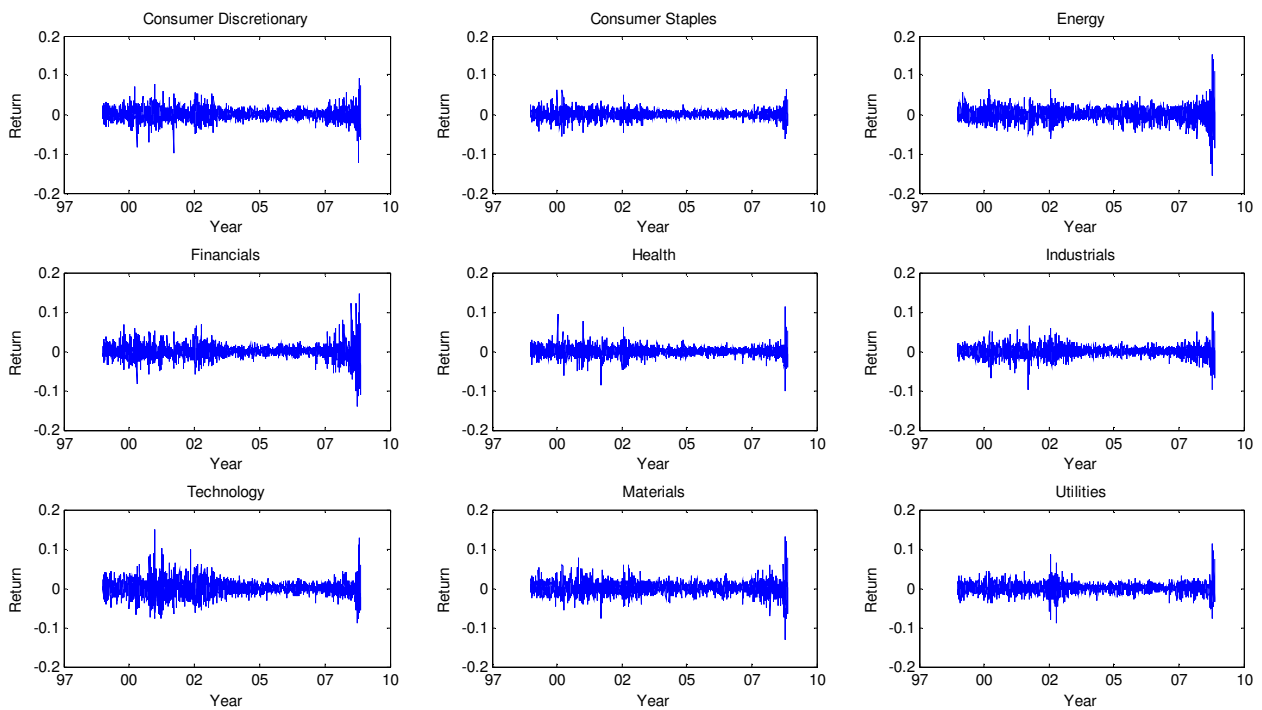

Figure 1 This figure plots the daily log returns of nine sector SPDR ETFs between from December 22, 1998 to November 17, 2008. The period from December 22, 1998 to March 10, 2003 features a high volatility due to burst internet bubbles and terrorist attacks, the second from March 11, 2003 to July 19, 2007 is of low volatility and the last one from July 20, 2007 to November 19, 2008 features an unprecedented high volatility due to the current financial crisis. In this paper, we choose the second period for applying our copula model to overnight and daytime returns.

\begin{tabular}{rrrrrrrrrrr}
\hline \hline & & XLY & XLP & XLE & XLF & XLV & XLI & XLB & XLK & XLU \\
\hline \multirow{6}{*}{ Overnight } & Mean & 0.0004 & 0.0004 & 0.0010 & 0.0009 & 0.0011 & 0.0007 & 0.0007 & 0.0004 & 0.0014 \\
& Std. Dev. & 0.005 & 0.004 & 0.006 & 0.005 & 0.005 & 0.005 & 0.005 & 0.007 & 0.005 \\
& Skewness & 0.675 & -0.350 & -0.056 & 0.506 & 1.318 & -0.214 & 0.046 & 0.084 & 0.428 \\
& Kurtosis & 9.810 & 5.533 & 4.051 & 7.589 & 29.841 & 6.229 & 7.421 & 7.191 & 7.712 \\
\hline \multirow{2}{*}{ Daytime } & Mean & 0.0002 & 0.00004 & 0.0002 & -0.0002 & -0.0007 & 0.0001 & 0.0002 & 0.0002 & -0.0005 \\
& Std. Dev. & 0.008 & 0.006 & 0.012 & 0.008 & 0.008 & 0.009 & 0.010 & 0.010 & 0.009 \\
& Skewness & 0.064 & 0.268 & -0.270 & -0.032 & 0.035 & 0.081 & -0.183 & -0.093 & -0.206 \\
& Kurtosis & 4.900 & 4.189 & 3.340 & 4.690 & 5.080 & 4.523 & 3.650 & 4.523 & 3.940 \\
\hline \hline
\end{tabular}

Table 1 This table reports the descriptive statistics (mean, standard deviation, Skewness and Kurtosis) for overnight and daytime returns of the SPDR ETFs of nine sectors between March 11, 2003 and July 19, 2007. We find overnight returns are all positive and consistently higher than daytime returns. Daytime returns have significantly higher standard deviations than overnight returns. Skewness for both returns has mixed signs and Kurtosis is greater than three for both returns and overnight returns tend to have higher Kurtosis. XLY, XLP, XLE, XLF, XLV, XLI, $\mathrm{XLB}, \mathrm{XLK}$, and XLU represent the sectors of Consumer Discretionary, Consumer Staples, Energy, Financial, Healthcare, Industrial, Materials, Technology and Utilities. 


\begin{tabular}{lcrrrrrrrrr}
\hline \hline & & XLY & XLP & XLE & XLF & XLV & XLI & XLB & XLK & XLU \\
\hline \multirow{2}{*}{ Overnight } & $\alpha_{i, 0}$ & $\mathbf{0 . 0 0 0 4}$ & $\mathbf{0 . 0 0 0 4}$ & $\mathbf{0 . 0 0 1 1}$ & $\mathbf{0 . 0 0 0 8}$ & $\mathbf{0 . 0 0 1 0}$ & $\mathbf{0 . 0 0 0 7}$ & $\mathbf{0 . 0 0 0 7}$ & $\mathbf{0 . 0 0 0 4}$ & $\mathbf{0 . 0 0 1 3}$ \\
& & 0.0001 & 0.0001 & 0.0002 & 0.0002 & 0.0001 & 0.0002 & 0.0002 & 0.0002 & 0.0002 \\
& $\alpha_{i, 1}$ & -0.03 & $\mathbf{- 0 . 0 5}$ & $\mathbf{0 . 0 7}$ & -0.01 & -0.02 & $\mathbf{0 . 0 4}$ & 0.01 & -0.02 & 0.01 \\
& & 0.02 & 0.02 & 0.01 & 0.02 & 0.02 & 0.02 & 0.02 & 0.02 & 0.02 \\
& $\alpha_{i, 2}$ & -0.01 & 0.0001 & $\mathbf{- 0 . 1 1}$ & 0.01 & 0.02 & $\mathbf{0 . 0 8}$ & -0.01 & -0.05 & $\mathbf{0 . 1 1}$ \\
& & 0.03 & 0.03 & 0.03 & 0.03 & 0.03 & 0.03 & 0.03 & 0.03 & 0.03 \\
\hline \multirow{2}{*}{ Daytime } & $\beta_{i, 0}$ & 0.0003 & 0.0002 & 0.0002 & 0.0001 & -0.0001 & 0.0005 & 0.0003 & 0.0004 & 0.0004 \\
& & 0.0002 & 0.0002 & 0.0004 & 0.0002 & 0.0002 & 0.0002 & 0.0003 & 0.0003 & 0.0003 \\
& $\beta_{i, 1}$ & $\mathbf{- 0 . 3 0}$ & $\mathbf{- 0 . 5 0}$ & -0.01 & $\mathbf{- 0 . 4 0}$ & $-\mathbf{0 . 5 5}$ & $-\mathbf{0 . 5 9}$ & $\mathbf{- 0 . 1 4}$ & $\mathbf{- 0 . 3 3}$ & $\mathbf{- 0 . 6 6}$ \\
& & 0.05 & 0.05 & 0.06 & 0.05 & 0.05 & 0.04 & 0.06 & 0.04 & 0.05 \\
& $\beta_{i, 2}$ & -0.01 & $\mathbf{- 0 . 1 0}$ & -0.05 & $\mathbf{- 0 . 0 8}$ & -0.04 & -0.04 & 0.0003 & -0.03 & -0.02 \\
& & 0.03 & 0.03 & 0.03 & 0.03 & 0.03 & 0.03 & 0.03 & 0.03 & 0.03 \\
\hline \hline
\end{tabular}

Table 2 This table reports the OLS estimates of conditional mean parameters for each ETF. The values in italics are standard errors. Estimates in bold are statistically significant at a 5\% confidence level. Interestingly, the constant terms are all statistically significant and positive. Not many overnight returns significantly depend on previous daytime and overnight returns. For significant ones, the signs are mixed. In contrast, most constant terms for daytime returns are not statistically significant. Almost all daytime returns statistically depend on previous overnight returns negatively. Few of them depend on their own lag values. 


\begin{tabular}{|c|c|c|c|c|c|c|c|c|c|c|}
\hline & & XLY & XLP & XLE & XLF & XLV & XLI & XLB & XLK & XLU \\
\hline \multirow[t]{10}{*}{ Overnight } & $\theta_{i, 0}$ & $1.36 \mathrm{E}-07$ & 7.01E-07 & 4.66E-08 & 3.69E-07 & 7.43E-07 & $1.78 \mathrm{E}-07$ & $1.69 \mathrm{E}-06$ & $1.15 \mathrm{E}-06$ & $2.72 \mathrm{E}-06$ \\
\hline & & $2.76 E-07$ & $2.37 E-07$ & $4.58 E-08$ & $3.35 E-07$ & $3.32 E-07$ & $2.95 E-07$ & $9.86 E-07$ & $9.90 E-07$ & $2.05 E-06$ \\
\hline & $\theta_{i, 1}$ & 0.024 & 0.026 & 0.004 & 0.017 & 0.026 & 0.025 & 0.008 & 0.032 & 0.008 \\
\hline & & 0.009 & 0.010 & 0.003 & 0.007 & 0.015 & 0.014 & 0.008 & 0.012 & 0.007 \\
\hline & $\theta_{i, 2}$ & 0.004 & 0.010 & 0.032 & 0.040 & 2.79E-09 & 0.029 & 0.043 & 0.026 & 0.058 \\
\hline & & 0.010 & 0.011 & 0.015 & 0.015 & $6.28 E-07$ & 0.015 & 0.019 & 0.027 & 0.044 \\
\hline & $\theta_{i, 3}$ & 0.922 & 0.883 & 0.947 & 0.904 & 0.905 & 0.910 & 0.863 & 0.879 & 0.813 \\
\hline & & 0.027 & 0.022 & 0.023 & 0.030 & 0.024 & 0.041 & 0.060 & 0.055 & 0.111 \\
\hline & $\boldsymbol{v}_{i, n}$ & 4.397 & 9.027 & 27.145 & 4.220 & 3.333 & 16.430 & 4.197 & 4.504 & 4.890 \\
\hline & & 0.402 & 1.958 & 15.470 & 0.368 & 0.164 & 7.360 & 0.343 & 0.432 & 0.488 \\
\hline \multirow[t]{10}{*}{ Daytime } & $\delta_{i, 0}$ & $7.21 \mathrm{E}-06$ & 2.08E-06 & $2.44 \mathrm{E}-06$ & $5.05 \mathrm{E}-06$ & $1.99 \mathrm{E}-06$ & $1.91 \mathrm{E}-06$ & $3.33 \mathrm{E}-05$ & $9.21 \mathrm{E}-07$ & $5.69 \mathrm{E}-06$ \\
\hline & & $4.12 E-06$ & $1.05 E-06$ & $1.66 E-06$ & $3.83 E-06$ & $2.52 E-06$ & $1.59 E-06$ & $2.31 E-05$ & $1.36 E-06$ & $3.87 E-06$ \\
\hline & $\delta_{i, 1}$ & 0.187 & 0.132 & 0.095 & 0.169 & 0.047 & 0.027 & 0.370 & 0.053 & 0.027 \\
\hline & & 0.101 & 0.070 & 0.045 & 0.133 & 0.085 & 0.032 & 0.201 & 0.058 & 0.027 \\
\hline & $\delta_{i, 2}$ & 0.055 & 0.040 & 0.033 & 0.036 & 0.053 & 0.041 & 0.019 & 0.027 & 0.099 \\
\hline & & 0.021 & 0.023 & 0.015 & 0.018 & 0.037 & 0.021 & 0.019 & 0.019 & 0.041 \\
\hline & $\delta_{i, 3}$ & 0.764 & 0.841 & 0.928 & 0.815 & 0.889 & 0.914 & 0.528 & 0.936 & 0.805 \\
\hline & & 0.099 & 0.062 & 0.028 & 0.098 & 0.112 & 0.053 & 0.274 & 0.056 & 0.097 \\
\hline & $v_{i, d}$ & 11.259 & 14.232 & 76.314 & 7.388 & 8.699 & 12.600 & 14.936 & 17.693 & 11.009 \\
\hline & & 3.384 & 5.592 & 44.370 & 1.477 & 1.888 & 4.441 & 5.320 & 8.732 & 2.926 \\
\hline
\end{tabular}

Table 3 This table reports the GARCH estimates of marginal distributions for individual assets. The values in italics are robust standard errors. All GARCH terms and DoF parameters are statistically significant, while ARCH terms have mixed statistical significance and signs.Estimates in bold are statistically significant at a 5\% confidence level. Except XLI, we find all the other ETFs have lower DoF parameters for overnight returns than for daytime returns, which is consistent with the observed higher Kurtosis of overnight returns. 

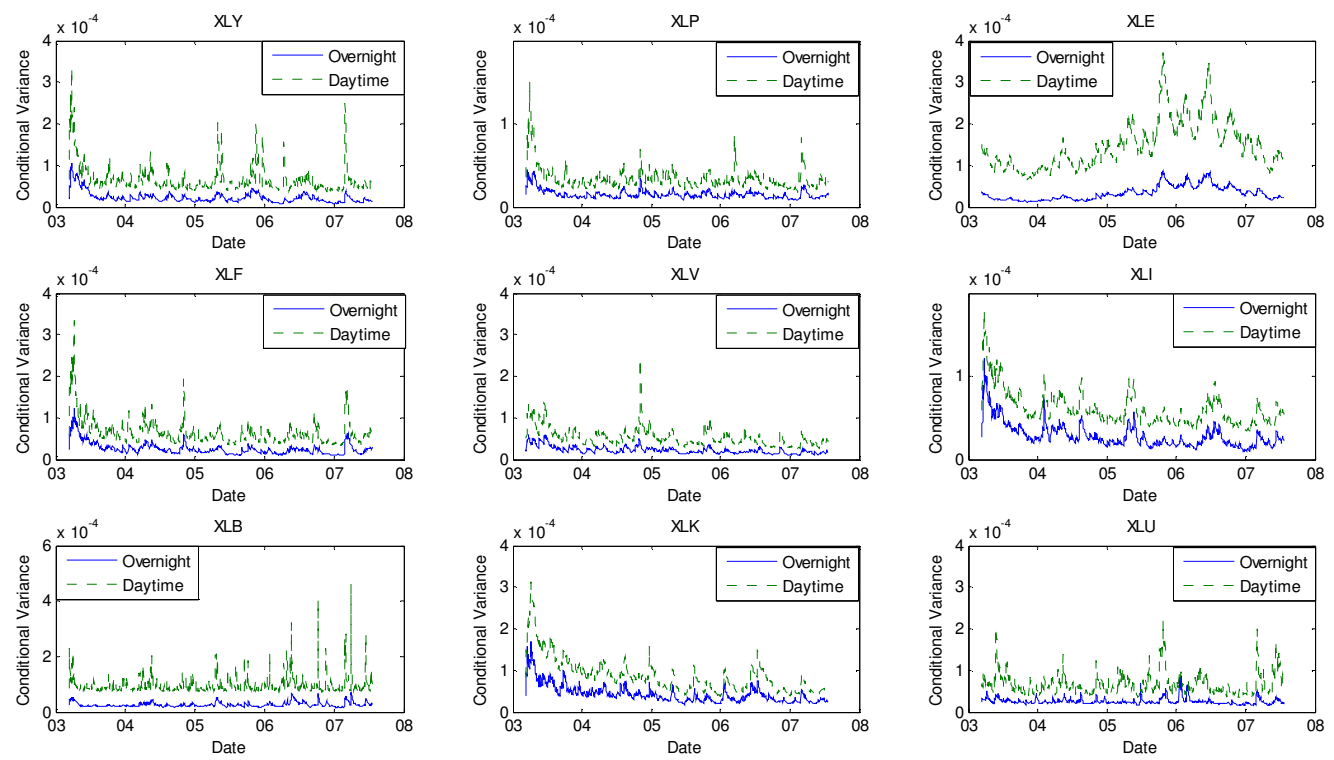

Figure 2 This figure plots the estimated conditional variance of overnight and daytime returns for each ETF returns. We find that the daytime returns have consistently higher conditional volatility than overnight returns. 


\begin{tabular}{|c|c|c|c|c|c|c|c|c|c|}
\hline \multicolumn{10}{|l|}{ Overnight } \\
\hline$R_{c n}$ & XLY & XLP & XLE & XLF & XLV & XLI & XLB & XLK & XLU \\
\hline \multicolumn{10}{|l|}{ XLY } \\
\hline XLP & 0.44 & & & & & & & & \\
\hline XLE & 0.18 & 0.18 & & & & & & & \\
\hline XLF & 0.57 & 0.42 & 0.22 & & & & & & \\
\hline XLV & 0.47 & 0.40 & 0.15 & 0.48 & & & & & \\
\hline XLI & 0.48 & 0.34 & 0.18 & 0.45 & 0.33 & & & & \\
\hline XLB & 0.47 & 0.37 & 0.25 & 0.48 & 0.35 & 0.40 & & & \\
\hline XLK & 0.55 & 0.37 & 0.18 & 0.54 & 0.40 & 0.44 & 0.48 & & \\
\hline XLU & 0.28 & 0.29 & 0.19 & 0.29 & 0.25 & 0.26 & 0.29 & 0.25 & \\
\hline$v_{c n}$ & 15.52 & & & & & & & & \\
\hline
\end{tabular}

\begin{tabular}{|c|c|c|c|c|c|c|c|c|c|}
\hline $\begin{array}{c}\text { Daytime } \\
R_{c d}\end{array}$ & XLY & XLP & XLE & XLF & XLV & XLI & XLB & XLK & XLU \\
\hline XLY & & & & & & & & & \\
\hline XLP & 0.68 & & & & & & & & \\
\hline XLE & 0.37 & 0.28 & & & & & & & \\
\hline XLF & 0.77 & 0.67 & 0.38 & & & & & & \\
\hline XLV & 0.64 & 0.63 & 0.32 & 0.65 & & & & & \\
\hline XLI & 0.77 & 0.64 & 0.43 & 0.73 & 0.62 & & & & \\
\hline XLB & 0.67 & 0.52 & 0.52 & 0.65 & 0.52 & 0.73 & & & \\
\hline XLK & 0.71 & 0.58 & 0.33 & 0.68 & 0.57 & 0.70 & 0.61 & & \\
\hline XLU & 0.46 & 0.45 & 0.45 & 0.52 & 0.41 & 0.48 & 0.49 & 0.41 & \\
\hline$\nu_{c d}$ & 24.01 & & & & & & & & \\
\hline Log-likelihood & 4786.96 & & & & & & & & \\
\hline
\end{tabular}

Table 4 This table reports the estimates for the two Student's t copulas governing the dependence structure of overnight and daytime returns. We find that overnight returns have lower values of correlation matrix and DoF parameters than daytime returns. We also report the log-likelihood for the copula components. 


\begin{tabular}{crr}
\hline \hline & Estimate & $\mathrm{SE}$ \\
\hline$\pi_{1}$ & 0.011 & 0.003 \\
$\pi_{2}$ & 0.005 & 0.002 \\
$\pi_{3}$ & 0.043 & 0.022 \\
$\pi_{4}$ & 0.920 & 0.027 \\
$\psi_{1}$ & 0.023 & 0.004 \\
$\psi_{2}$ & 0.009 & 0.008 \\
$\psi_{3}$ & 0.217 & 0.166 \\
$\psi_{4}$ & 0.290 & 0.232 \\
$\nu_{c n}$ & 15.177 & 1.898 \\
$\nu_{c d}$ & 25.071 & 3.835 \\
\hline & & \\
\hline Log-likelihood & 4863.84 & \\
\hline \hline
\end{tabular}

Table 5 This table shows the ML estimates for the time-varying t copula. Consistent with the constant case, the copula DoF parameter of daytime returns is higher than that of overnight returns. We also report the log-likelihood of the copula component. Compared with constant $t$ copula, we have significantly higher log-likelihood by making correlation matrices time-varying. 

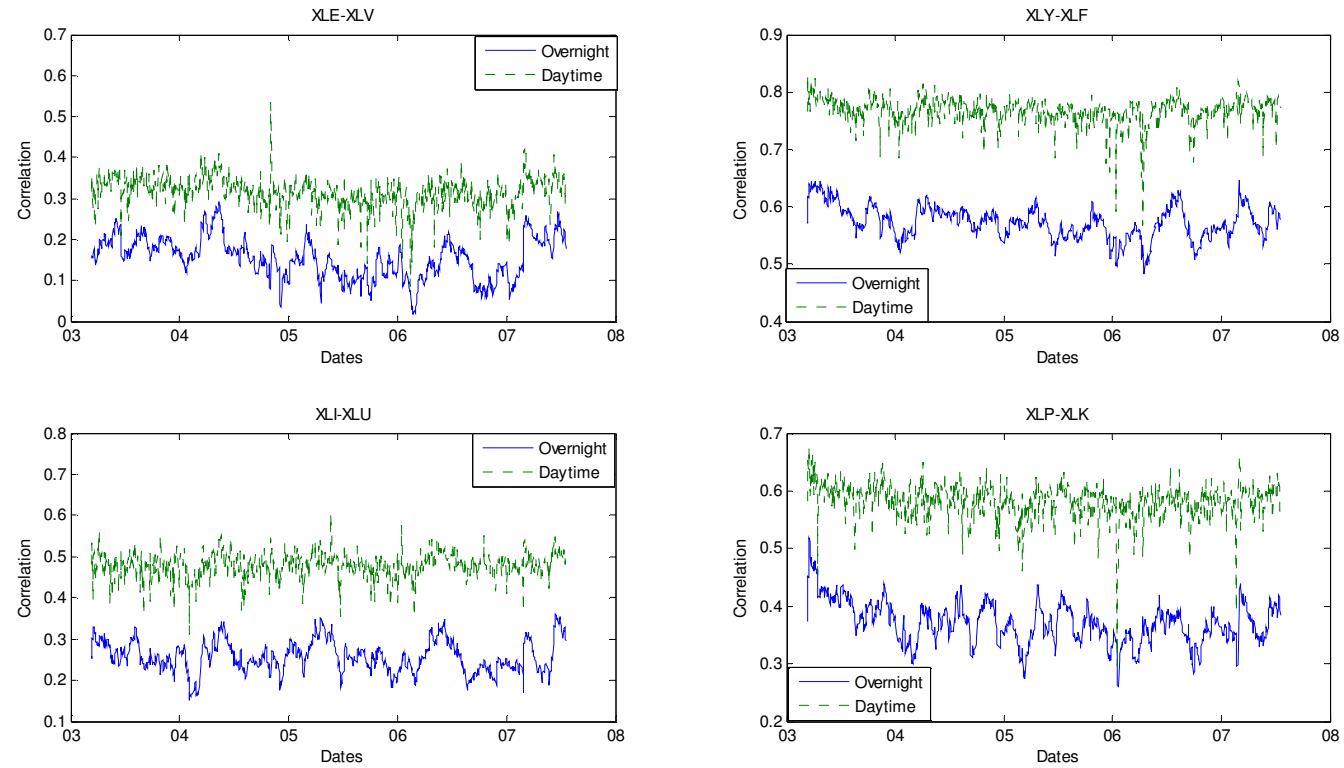

Figure 3 This figure plots the time-varying conditional correlation parameters of the $t$ copulas for four pairs of the returns. We observe that the correlation parameter of daytime returns is consistently higher than that of overnight returns.
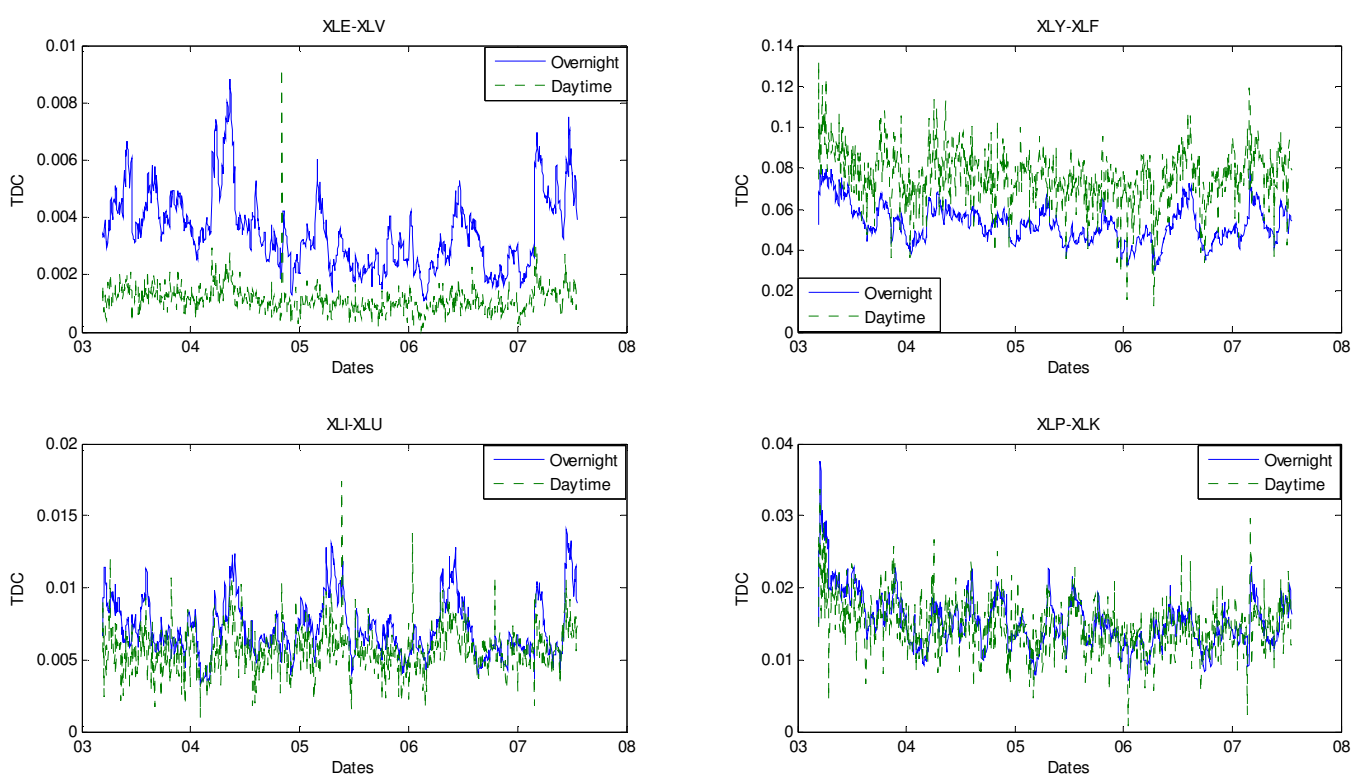

Figure 4 This figure plots the time-varying conditional tail dependence coefficient (TDC) for the four pairs of returns. We observe mixed patterns. 